\title{
Téoros
}

Revue de recherche en tourisme

\section{Le tourisme sexuel}

Ses plaisirs et ses dangers

\section{Joseph J. Lévy et Élyzabeth Lacombe}

Volume 22, numéro 1, printemps 2003

Tourisme et sexualité

URI : https://id.erudit.org/iderudit/1071578ar

DOI : https://doi.org/10.7202/1071578ar

Aller au sommaire du numéro

Éditeur(s)

Université du Québec à Montréal

ISSN

0712-8657 (imprimé)

1923-2705 (numérique)

Découvrir la revue

Citer cet article

Lévy, J. J. \& Lacombe, É. (2003). Le tourisme sexuel : ses plaisirs et ses dangers. Téoros, 22(1), 4-9. https://doi.org/10.7202/1071578ar

Ce document est protégé par la loi sur le droit d'auteur. L'utilisation des services d’Érudit (y compris la reproduction) est assujettie à sa politique d'utilisation que vous pouvez consulter en ligne.

https://apropos.erudit.org/fr/usagers/politique-dutilisation/
Cet article est diffusé et préservé par Érudit.

Érudit est un consortium interuniversitaire sans but lucratif composé de l’Université de Montréal, l’Université Laval et l'Université du Québec à Montréal. Il a pour mission la promotion et la valorisation de la recherche. https://www.erudit.org/fr/ 


\section{Le tourisme sexuel Ses plaisirs et ses dangers}

\section{Joseph J. Lévy et Élyzabeth Lacombe}

e tourisme sexuel constitue un secteur important dans la configuration des voyages contemporains et il présente des formes variées en fonction des régions et des cultures. De plus, il contribue de façon significative à la transmission des infections transmises sexuellement et du $\mathrm{VIH} /$ sida. Cet article esquisse les principaux enjeux liés à ces problématiques ainsi que les réponses nationales et internationales visant à contrôler et à gérer le développement de cette forme de tourisme dans le contexte de la mondialisation.

Le tourisme, qui entraîne aujourd'hui le déplacement annuel de près de 600 millions de personnes, est l'un des secteurs économiques en pleine expansion, contribuant significativement aux budgets nationaux de nombreux pays. Dans les représentations collectives modernes associées au tourisme, les quatre $\mathrm{S}-\mathrm{Sun}$, Sex, Sea and Sand - permettent d'échapper pour un temps aux contraintes qu'une société hautement technologique exerce sur l'individu. La détente, l'évasion, la liberté constituent ainsi des motifs centraux dans les motivations touristiques (Krippendorf, 1987). Dans le contexte liminoïde offert par ce séjour (Lett, 1983), les touristes sont à même d'expérimenter les dimensions davantage ludiques de son existence (Cohen et Taylor, 1976 ; Ryan, 1991) et de rechercher des sensations fortes associées à la consommation d'alcool et de drogues. Ils peuvent alors vivre des sensations, nouvelles et complexes tout en étant prêts à prendre les risques nécessaires pour les obtenir.

Certaines recherches ont ainsi mis en évidence un lien entre la quête de sensations fortes et les voyages internationaux (Mayo et Jarvis, 1981) pendant lesquels les touristes expérimentent un changement dans leurs états de conscience associés aux contrastes entre les activités touristiques et les expériences familières dans le pays d'origine. De ce point de vue, la structure du voyage touristique se rapproche de celle des rituels (Graburn, 1983) en créant une rupture spatio-temporelle particulière qui peut s'accompagner d'une quête de type religieux à travers les expériences alternatives nouvelles et inhabituelles, ou plus existentielles, qui peuvent inclure des expériences érotiques.

Les nouvelles relations sociales, nouées dans un cadre où les normes habituelles de comportements sont mises entre parenthèses, peuvent en effet s'accompagner de rencontres d'occasions sexuelles, romantiques ou physiques entre les touristes eux-mêmes, ou entre les touristes et des personnes des pays d'accueil (Ryan, 1991; Graburn, 1983). Les motivations du voyage peuvent par ailleurs être plus directement sexuelles et s'inscrire dans le cadre d'un tourisme sexuel, en progression, défini comme « un tourisme où l'objectif essentiel de la motivation est de consommer des relations sexuelles commerciales » (Hall, 1992 : 64) associées aux différentes formes de prostitution. Le degré d'exploitation sexuelle dépend des régions géographiques et des pays dont certains constituent de véritables «pornocraties » (Manderson, 1992).

\section{Le tourisme sexuel et ses plaisirs}

Dans certains cas, la publicité touristique vante de façon détournée les promesses et le potentiel des destinations sexuelles et tente d'attirer les vacanciers vers des destinations exotiques en décrivant les paysages dans un langage suggestif, les assimilant à des femmes qui, lorsqu'elles sont représentées, sont associées à la passivité, à la soumission et à la séduction. Le langage touristique refléterait ainsi une idéologie patriarcale axée sur les normes hétérosexuelles (Pritchard et Morgan, 2000), bien que le tourisme gai contribue à modifier cette perspective. À d'autres occasions, la publicité sur la prostitution masculine ou féminine est plus directe, en particulier dans le cas des destinations les plus sexuelles. L'accroissement du tourisme a ainsi favorisé la prostitution féminine et masculine qui fournit des sources de revenus essentiels ou d'appoint dans les régions à haute densité touristique.

Plusieurs recherches ont montré que le développement rapide du tourisme dans certaines régions s'accompagnait de l'extension de formes multiples de prostitution qui, dans des contextes où les difficultés économiques prédominent, permettent d'assurer un accès à des ressources matérielles en échange de faveurs sexuelles. $\mathrm{La}$ prostitution constitue dans plusieurs contextes une stratégie de survie, accélérant l'exploitation, sinon l'esclavage sexuel des femmes et des jeunes garçons et filles.

La prostitution serait la plus élevée dans les pays asiatiques comme la Thaïlande, les Philippines, l'Indonésie et la Malaisie où elle contribuerait significativement au pro- 


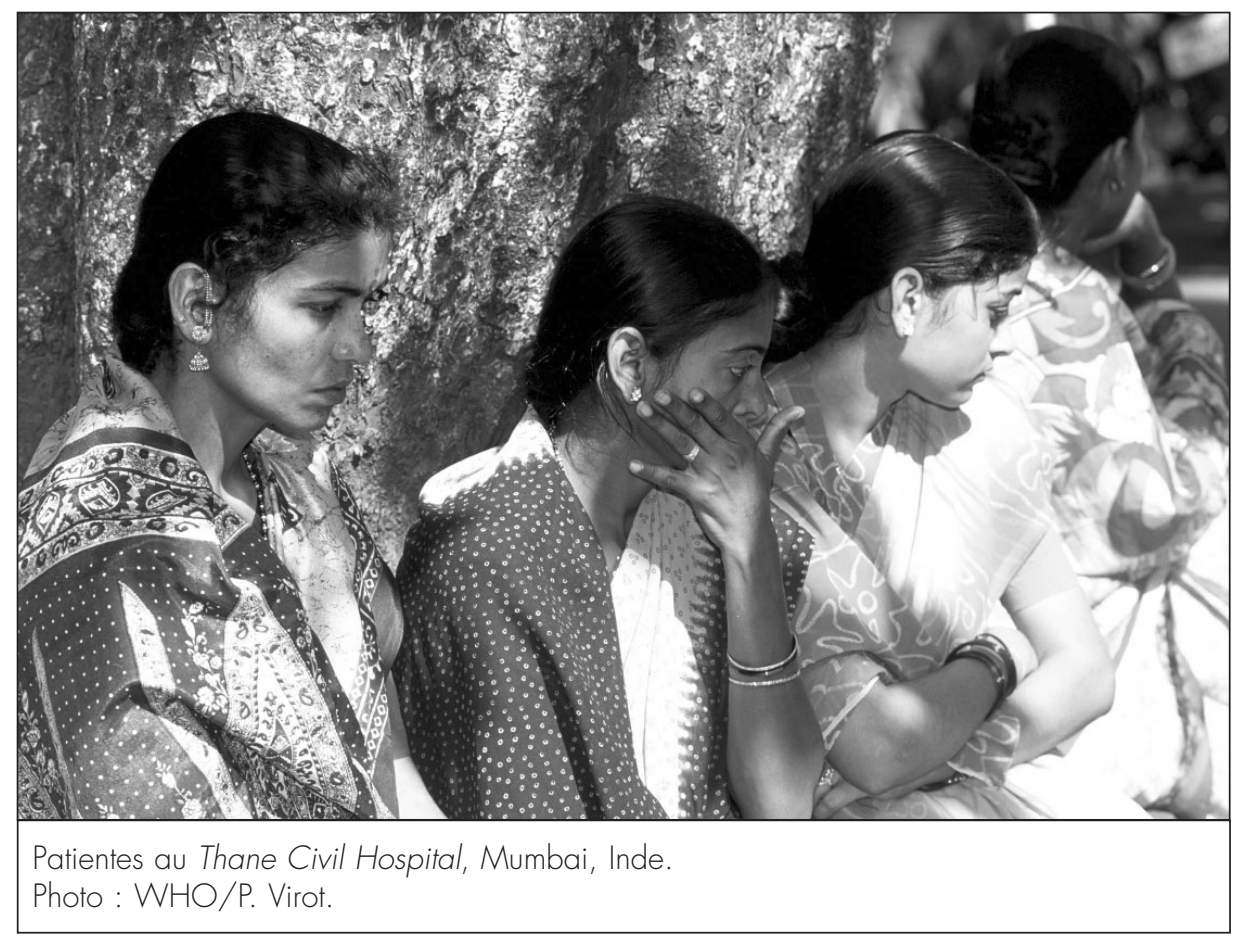

duit intérieur brut (de $2 \%$ à $14 \%$ ). L'Amérique latine (Brésil, Colombie, Costa Rica) et les Caraïbes (Cuba, République dominicaine) sont aussi des zones de haute densité eu égard au nombre de prostitués, en particulier des femmes. En Afrique, la zone maghrébine, la Zambie, le Kenya et Madagascar sont des pays où l'on assiste à une progression de la prostitution.

Il existe cependant des variations importantes selon les régions, les modes de recrutement, les catégories de prostitués, les échanges économiques et le contexte institutionnel dans lequel ces relations prennent place. L'un des cas les plus connus est celui de la Thailande où les normes sexuelles se fondent sur un double standard sexuel. Les hommes - toutes catégories sociales confondues, qu'ils soient célibataires ou mariés - sont habitués à fréquenter les prostituées, ce qui constituerait un marqueur de leur masculinité. Le trafic de jeunes garçons ou de jeunes filles - souvent à peine pubères - enlevés et vendus à des proxénètes moyennant argent ou biens matériels, sert à alimenter les lieux de prostitution. Les prostituées indépendantes, call-girls ou « nolisées » (O’Malley, 1988) dans certains cas, établissent des relations davantage affectives avec les touristes (Cohen, 1982). Les services sexuels peuvent aussi être offerts dans le cadre de voyages sexuels or- ganisés où des jeunes filles ou des jeunes garçons répondent aux demandes sexuelles des touristes. Au Mexique (Carrier, 1989), la prostitution, un phénomène encore mal cerné, recouvre des catégories multiples qui vont des prostituées de rue aux escortes. Ainsi, à Cuba, chômeuses et étudiantes, que l'on désigne sous le nom de jineteras, servent d'escorte aux touristes en échange d'argent ou de biens, une fonction qui peut conduire à l'adoption plus complète de la prostitution.

La prostitution masculine est aussi l'une des formes présentes dans plusieurs pays, soit sous la forme d'exploitation des enfants et des adolescents par des homosexuels, soit sous la forme d'une institutionnalisation de rapports d'hommes adultes avec des touristes de sexe féminin (qui pourraient constituer près de $10 \%$ du contingent des femmes touristes selon Maurer, 1992). Au Moyen-Orient (Cohen, 1971), les boutiquiers peuvent accompagner leurs marchandages avec les touristes américaines et européennes de demandes sexuelles. En Grèce, les prostitués masculins - les $\mathrm{ka}$ makia (ou harpons) - peuvent même former des associations et émettre des cartes de séronégativité. En Gambie, des femmes suédoises d'âge mûr entretiennent des relations sexuelles avec des jeunes hommes qu'elles ramènent quelquefois avec elles. Les play- boys professionnels ou les beach boys constituent une forme particulière de prostitués, utilisée surtout par des femmes en quête d'expériences sexuelles, que l'on trouve dans les régions caraibe, africaine et asiatique. Généralement travailleurs autonomes, ils œuvrent souvent dans le secteur du tourisme comme guides, employés d'hôtels, en particulier dans l'animation, ce qui leur permet d'avoir des contacts faciles avec les voyageurs (Forsythe et al., 1998 ; Dahles et Bras, 1999). Polyglottes, ils ont généralement moins de 25 ans (Herold et al., 2001). Ils portent une attention particulière à leur apparence physique, soignent leur image, notamment en accentuant leurs caractéristiques exotiques (Dahles et Bras, 1999 ; Herold et al., 2001). Non seulement ils ne cachent pas leurs activités, mais ils insistent pour être vus en public avec leurs clientes, ce qui leur procurerait un certain statut auprès de leurs confrères (Herold et al., 2001). En Tunisie (Lévy et al., 2001), les dragueurs, ou bezness selon la terminologie locale, rendent des services sexuels aux touristes et se font payer selon des modalités diverses (argent, dons monétaires ou cadeaux) qui constituent quelques foois leur seul revenu, mais l'établissement de relations affectives ou purement sexuelles, sans retour matériel, est aussi présent. Les dragueurs peuvent passer d'une forme à l'autre, en fonction des contraintes variables (expérience, saison, type de partenaire).

En Jamaïque (Pruitt et Lafont, 1995), les beach boys développent des stratégies complexes pour obtenir les faveurs sexuelles et pécuniaires des touristes, le plus souvent des femmes. En République dominicaine, dans les années soixante-dix et quatre-vingt, les beach boys avaient surtout des relations sexuelles avec des hommes, une tendance qui a changé dans les années quatre-vingtdix, les touristes sexuels étant de plus en plus fréquemment des femmes. Ils choisiront leur cible en fonction de leur but : s'il s'agit d'une conquête sexuelle ou amoureuse, les touristes seront généralement jeunes et attirantes. Dans le cas où c'est l'intérêt économique qui prime, elles seront sélectionnées parmi les touristes plus âgées ou n'obéissant pas aux canons de beauté physique dominants, ce qui augmenterait leur 
vulnérabilité aux avances du beach boy (Herold et al., 2001). En Indonésie, les beach boys vont préférer avoir pour clientes des jeunes femmes de leur âge ; toutefois, ils peuvent aussi choisir des femmes plus âgées, pensant qu'elles sont mieux nanties financièrement (Dahles et Bras, 1999).

Les voyageurs qui utilisent des services sexuels présentent aussi des profils tout à fait hétérogènes, en termes de nationalité, de genre, d'âge, d'origine ethnique, d'orientation sexuelle, de statut socio-économique, de pratiques sexuelles et de significations qu'ils accordent à ces rencontres (O'Connell Davidson, 2001). Deux grands types de touristes sexuels ont pu être dégagés : " les touristes sexuels occasionnels » qui voyagent pour des raisons non sexuelles et qui pourront, à l'occasion, avoir des relations sexuelles avec des travailleurs du sexe et les « touristes sexuels assidus » ou hard-core qui ne voyagent que dans le but d'avoir des relations sexuelles, souvent interdites dans leur pays d'origine. Dans la première catégorie on trouve des hommes, mais aussi de plus en plus de femmes alors que les touristes sexuels hard-core seraient surtout des hommes, d'origine caucasienne. La majorité des touristes ne voyagerait pas avec l'intention expresse d'avoir des relations sexuelles en vacances (Oppermann, 1999), ce que confirment Forsythe et al. (1998) à la suite d'une étude sur le tourisme sexuel en République dominicaine ainsi que Clift et Forrest (1999) qui ont étudié une population d'hommes gais. Toutefois, Oppermann (1999), à partir d'une revue des études sur ce sujet, suggère que le nombre de voyageurs s'adonnant au tourisme sexuel serait en fait plus élevé que ce que les études révèlent, car peu de gens accepteraient l'étiquette de " touriste sexuel », même si leurs activités durant les vacances pourraient les classer dans cette catégorie. Celles-ci s'inscriraient dans le répertoire érotique habituel ou, au contraire, constitueraient un moyen d'exploration de conduites plus rares. Ainsi, en Tunisie (Lévy et al., 2001), selon les bezness, les touristes seraient enclins à s'adonner à des formes de sado-masochisme, de voyeurisme et d'exhibitionnisme et à l'utilisation de gadgets ou d'objets sexuels.

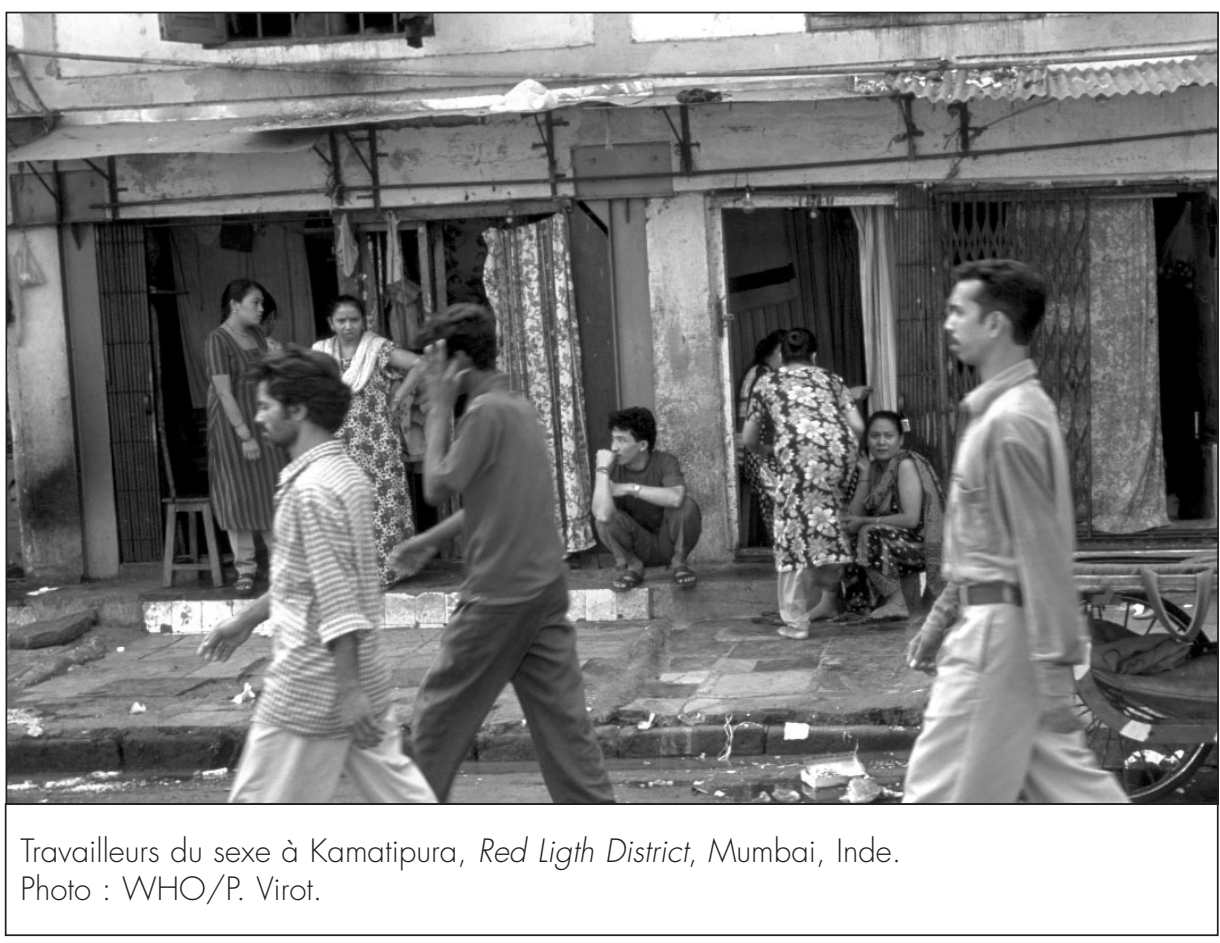

Les hard-core constitueraient la minorité et choisiraient leur destination en fonction des possibilités perçues d'avoir des relations sexuelles associées à des expériences particulières qu'ils pourraient difficilement vivre chez eux (relations impliquant plusieurs partenaires) ou encore avec des partenaires spécifiques (mineurs, transsexuels, etc.) (O'Connell Davidson, 2001), en particulier dans un contexte de pédophilie lié à la quête de la transgression d'interdits ou à la recherche d'une puissance sexuelle renouvelée. Certains touristes sexuels apprécient tellement leurs expériences qu'ils décident de venir vivre plus complètement dans le pays, perçu comme un paradis sexuel. C'est ce que O'Connell Davidson (2001) définit comme des « sexpatriés » (sexpatriates) installés en République dominicaine où ils participent par la suite à l'organisation du tourisme sexuel sur l'île. Ils se constituent ainsi en communautés où ils s'entraident pour accéder aux expériences sexuelles désirées, en s'échangeant des conseils, des renseignements, etc. Pour devenir membre de cette communauté, il suffit d'avoir une relation sexuelle avec une Dominicaine et de raconter ses ébats aux autres membres du groupe, ce qui constituerait une forme de rituel initiatique. Il existe aussi des clubs exclusifs, par exemple le Travel and the Single Male (TSM), qui ont leur propre portail Internet où les membres peuvent ob- tenir notamment de l'information sur le tourisme sexuel et participer à des chats (clavardages) pour échanger des conseils sur différentes destinations (O'Connell Davidson, 2001). Le profil sociopolitique de ces touristes se rapprocherait de ceux des groupes « suprémacistes ». Ils considèrent ainsi que leurs droits sont brimés dans leur pays d'origine où ils doivent composer avec l'autorité qui ne reconnaît pas assez leur supériorité en tant qu'homme blanc et impose des contraintes quant à l'expression de leur sexualité. Ces contraintes entreraient en conflit avec leurs « droits naturels », tels l'accès libre aux femmes qu'ils traiteront comme des objets, s'affirmant ainsi en tant qu'hétérosexuels dominants. Pour ces hommes, les voyages hors Amérique du Nord ou Europe représenteraient une façon de se défaire du stress inhérent à l'homme blanc dans les sociétés post-modernes contemporaines (O'Connell Davidson, 2001).

Le tourisme peut aussi comprendre d'autres services à connotation sexuelle ou érotique directe, comme les salons de massages et les saunas, ou plus indirecte, comme les boîtes de nuit et les discothèques. Les clubs de strip-tease constituent aussi à cet égard, selon Ryan et Martin (2001), un contexte hautement symbolique et ritualisé d'expression du tourisme sexuel qui renvoie 
à une performance publique où l'homme peut observer le corps féminin nu sans l'accompagner du contact sexuel impliqué dans le recours à la prostitution, tout en maintenant cependant un rapport personnalisé limité. En ce sens, le strip-tease est une activité de type « invitationnel », «mimant le désir mais sans sa satisfaction finale » (Ryan et Martin, 2001 :155) et inversant les rôles socio-sexuels en plaçant les femmes, pour un bref moment, dans une position sociale supérieure. Des spectacles publics, plus directement pornographiques, basés sur un voyeurisme plus prégnant, sont aussi prisés par les touristes, comme c'est le cas en Thaillande (Manderson, 1992). Ils comprennent des représentations mimant des activités sexuelles individuelles (mettant en relief les capacités de contrôle des muscles péri-vaginaux), en couple (relations hétérosexuelles et lesbiennes) ou en trio, qui ont pour objectif à la fois de maximiser les fantasmes érotiques et de servir l'expression satirique des clients présents.

Le tourisme gai (Clift et Forrest, 1999) constitue par ailleurs un marché en plein développement et de plus en plus de sites et de guides orientés vers ces populations mettent en perspective les destinations, les lieux, les services et les événements associés au monde gai. Bien que les études sur le tourisme gai soient encore rares, elles mettent en relief l'importance des rencontres sexuelles comme l'une des motivations du voyage chez certains hommes, bien que celles associées aux aspects culturels ou à la détente ne soient pas absentes. Ces différentes motivations interviendraient sur le choix des destinations.

\section{Le tourisme sexuel et ses dangers}

Le développement du tourisme sexuel n'est pas sans avoir des conséquences profondes sur l'état de santé mentale et physique des personnes qui sont impliquées dans les différentes activités de prostitution. Ainsi, le rapport d'exploitation, sinon d'esclavage, s'accompagne d'un degré de violence élevé et de formes de dépression profonde qui peuvent aller jusqu'au suicide. L'emploi de drogues et d'alcool est aussi répandu, ce qui contribue au délabrement mental associé aux symptômes de stress post-traumatique particulière- ment sévères (Farley et al., 1998). Dans les sociétés où les normes sexuelles dominantes sont peu permissives, le statut social problématique des prostitués peut entraîner leur stigmatisation et leur exclusion des réseaux sociaux et familiaux, d'où une marginalisation poussée. C'est le cas, par exemple, en Tunisie, où les bezness se trouvent coincés entre des modèles socioculturels contradictoires qui peuvent entraîner des sentiments d'aliénation personnelle et sociale, ce qui affecte leur bien-être (Lévy et al., 2001).

Sur le plan physique, les maladies gynécologiques, la tuberculose et l'hépatite C, mais surtout la transmission des infections sexuellement transmises et du VIH/sida sont en progression. Selon Rodriguez-Garcia (2001), la multiplication exponentielle des voyages internationaux s'est accompagnée d'une augmentation énorme de ces infections qui sont passées d'une estimation de 100000 cas en 1980 à 20 millions en 1993. Une grande proportion des cas est attribuable aux échanges sexuels liés au tourisme. La transmission du VIH/sida obéit aux mêmes tendances et les études épidémiologiques indiquent une forte corrélation entre les cas de séropositivité et de sida et l'importance du tourisme. L'étude de Nossa et al. (1994) au Portugal confirme ainsi qu'il existe une corrélation entre les patterns de distribution des cas de sida et les zones touristiques.

Cette situation dramatique s'explique par un ensemble de facteurs dégagés par Forsythe et al. (1998). Selon ces auteurs, la fréquence des relations sexuelles entre les voyageurs et entre ces derniers et les populations locales, particulièrement chez les jeunes, est élevée et les relations s'accompagnent d'un usage pour le moins arbitraire du préservatif, une situation à laquelle la consommation d'alcool importante peut contribuer. Ainsi, selon une étude effectuée en Angleterre (Ford, 1990 ; 1991 ; Ford et Inman, 1992), le quart des touristes interrogés avaient eu des relations sexuelles avec une personne qu'ils venaient de rencontrer, soit avec d'autres touristes, soit avec des personnes locales. Parmi celles qui travaillaient dans le secteur touristique, le nombre des partenaires sexuels occasionnels était plus élevé que dans le reste de la population, tout comme la consommation de drogues et d'alcool. Daniels et al. (1992), quant à lui, a montré que les relations sexuelles non protégées chez des touristes internationaux britanniques étaient plus présentes chez les femmes que chez les hommes, ce qui pouvait contribuer significativement à la transmission des ITS ou du VIH/sida.

D'autres études sur les touristes sud-coréens (Choi et al., 1992) indiquent que, bien que ceux-ci soient au courant des modalités de transmission du virus du sida, ils sous-estiment les risques liés aux contacts sexuels lors de leurs voyages et ont des comportements sexuels à haut risque. On trouve aussi ce type de conduite chez les touristes japonais visitant la Thailande ; ceux-ci avaient un haut taux d'utilisation du préservatif lors de leur première rencontre avec des prostituées, mais cet usage diminuait significativement lors des visites suivantes (Vorakitphokatorn et Cash, 1992 ; Cash, 1994). Parmi les touristes allemands qui se définissaient comme des touristes sexuels (Kleiber et al., 1995), l'usage du préservatif variait en fonction des destinations, passant de $30 \%$ dans le cas des Philippines, à $43 \%$ au Kenya, à $50 \%$ en Thailande, à $53 \%$ au Brésil et à $75 \%$ en République dominicaine. Dans ce dernier contexte, les touristes avaient tendance à surévaluer la prévalence des cas de séropositivité dans le pays, mais continuaient néanmoins à avoir des relations sexuelles non protégées.

Les études récentes menées en République dominicaine sur un échantillon de travailleuses du sexe (Forsythe et al., 1998) indiquaient que les touristes employaient fréquemment le préservatif dans leurs activités sexuelles avec elles, mais que cet usage était beaucoup moins fréquent dans leurs relations avec des partenaires locaux. Les voyageurs et les touristes visitant Hong Kong avaient aussi des relations sexuelles avec des prostituées sans utiliser, le plus souvent, une protection adéquate, concurremment à un usage immodéré d'alcool (Abdullah et al., 1996).

Les relations homosexuelles des touristes avec des prostitués s'accompagnent aussi de pratiques à risques, comme c'est le cas à Bali (Ford et al., 1995). Les touristes de 


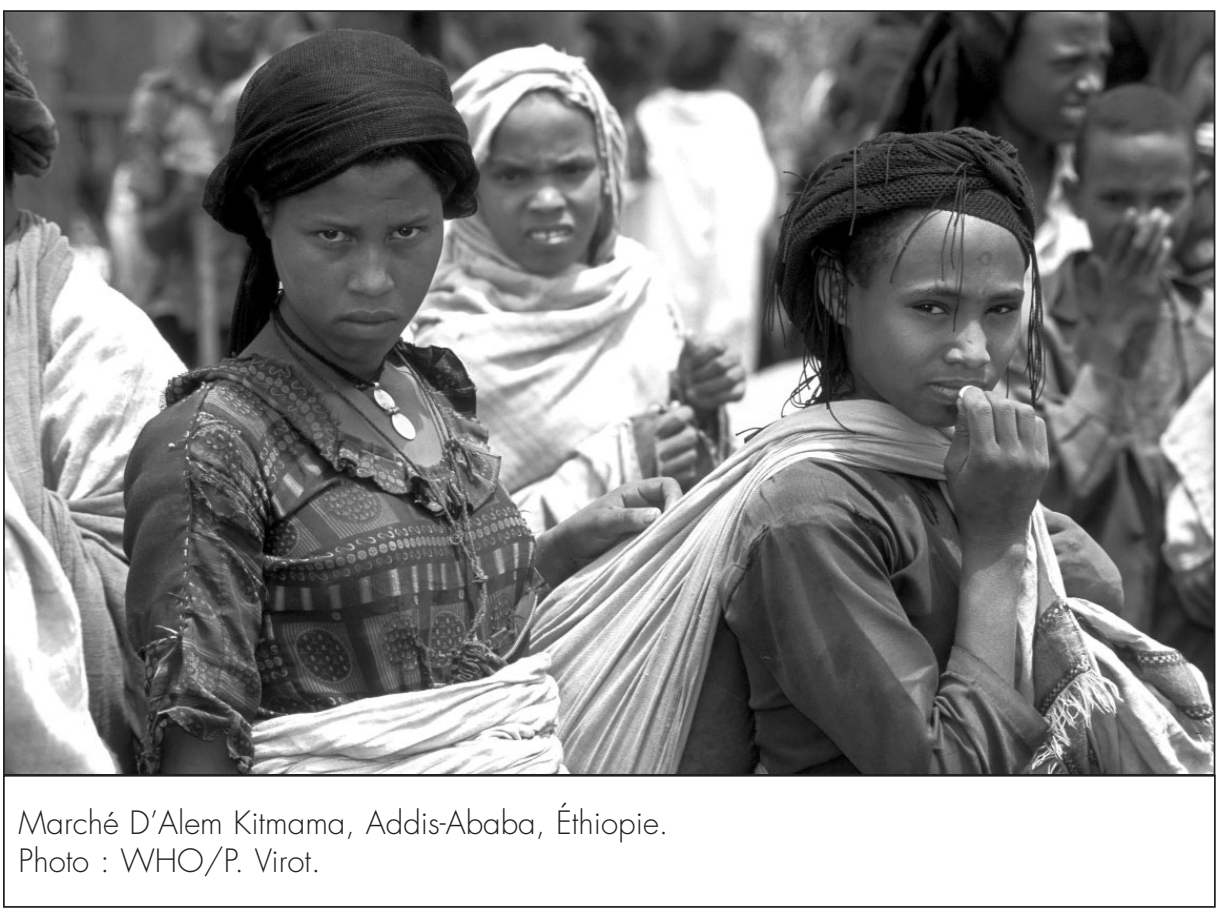

sexe féminin, occidentales et non occidentales, semblent aussi s'impliquer dans des activités sexuelles sans protection. C'est le cas des jeunes femmes japonaises visitant la Thaïlande qui, étant peu au courant des stratégies de prévention, développent des relations occasionnelles avec le personnel local sans se protéger adéquatement (Vorakiphokatorn, 1994).

Dans l'ensemble donc, les carences dans les stratégies de protection restent encore élevées, une situation qui s'explique, entre autres, par les conceptions locales des modes de transmission, par les normes et les valeurs sexuelles entourant l'usage du préservatif comme gênant le déroulement des relations et le plaisir ressenti), par les rapports de pouvoir existant entre les partenaires et par les motivations financières qui peuvent favoriser le rejet du préservatif.

\section{Conclusion}

Le tourisme, et en particulier le tourisme sexuel, ne sont pas sans avoir des conséquences socioculturelles, psychologiques et médicales significatives à la fois pour les populations d'accueil et pour les voyageurs, ce que les dynamiques économiques liées à la mondialisation ne feront qu' amplifier dans l'avenir. Le développement des réseaux de prostitution internationaux rattachés au trafic des femmes et des enfants ainsi que les carences dans les systèmes de protection juridique et sociale de ces populations défavorisées posent des problèmes complexes qui nécessitent la coopération des États et la mise en place de politiques hautement synchronisées, à la fois par les institutions internationales gouvernementales et non gouvernementales.

Il existe déjà des outils internationaux, par exemple la Convention internationale des Nations unies pour les droits de l'enfant établie le 20 novembre 1989, qui, si elle était véritablement appliquée, pourrait aider à réduire l'exploitation sexuelle des enfants, à condition que les sanctions pénales soient appliquées tant localement qu'exterritorialement. Un protocole facultatif portant sur la vente d'enfants, la prostitution des enfants et la pornographie mettant en scène des enfants est aussi entré en vigueur en 2002. De nombreux organismes ont par ailleurs développé des stratégies pluridimensionnelles pour lutter contre ce phénomène, entre autres en organisant le premier Congrès mondial contre l'exploitation sexuelle des enfants à des fins commerciales qui s'est tenu à Stockholm en 1996 et en mobilisant les différentes instances qui font partie de la chaîne touristique, depuis les professionnels sur le terrain jus- qu'aux administrations étatiques et internationales.

En ce qui concerne la prostitution, la Convention des Nations unies promulguée en 1949 et portant sur la répression de la traite des être humains et de l'exploitation de la prostitution d'autrui et la Convention des Nations unies contre le crime organisé transnational promulguée en 2000 servent d'armature légale contre le proxénétisme, sans lutter directement contre la prostitution, une question que les organismes internationaux comme l'Organisation internationale du travail préfèrent approcher de façon pragmatique en reconnaissant, en réglementant et en taxant l'industrie du sexe, alors que l'Organisation mondiale de la santé préconise la légalisation et la décriminalisation de cette industrie afin de réduire la progression de l'épidémie du VIH/sida. Mais est-il possible de commencer à trouver réponses à ces questions sans remettre en question les rapports économiques inégalitaires entre le Nord et le Sud, les relations de pouvoir entre hommes et femmes ?

Dans le contexte épidémiologique actuel, comme nous l'avons vu, le tourisme contribue significativement à la diffusion du virus à la fois dans les pays d'accueil et ceux d'où proviennent les voyageurs. À cet égard, il semble que se dessine une volonté d'intervenir plus directement sur le plan du secteur touristique lui-même, afin de mettre en place des interventions visant à informer les touristes et les nationaux des mécanismes de transmission des ITS et du VIH/sida, de rendre plus disponibles préservatifs et seringues, de sensibiliser les populations les plus directement impliquées dans le travail du sexe et de les habiliter à négocier des pratiques sexuelles protégées, tout en leur assurant des salaires plus décents. Cependant, ces stratégies ne peuvent faire l'économie d'une analyse critique du tourisme en général, de ses motivations et de ses objectifs actuels pour développer un projet à même de réduire le déséquilibre économique et socioculturel actuel qu'une mondialisation des échanges mal menée risque d'amplifier.

Joseph J. Lévy est anthropologue et professeur au département de sexologie de l'Université du Québec à Montréal. Ses in- 
térêts de recherche portent sur les scénarios sexuels et la prévention des infections transmises sexuellement et du VIH/sida.

\section{Élyzabeth Lacombe est détentrice} d'un baccalauréat en criminologie de l'Université de Montréal et d'une maîtrise en sexologie de l'Université du Québec à Montréal (profil recherche). Elle est chargée de cours au Département de sexologie de l'UQAM et chargée de projet sur la prévention de l'homophobie en milieu de la jeunesse à la Direction de la santé publique de Montréal-Centre.

\section{Bibliographie}

Abdullah, A.S., A.J. Hedley, et R. Fielding (1996), « The Sexual Behaviour of Tourists in Hong Kong: A Pilot Study », International Conference on AIDS, 7-12 juillet, 173. Résumé $\mathrm{n}^{\mathrm{O}}$ Mo.D.1695.

Carrier, J.M. (1989), « Sexual Behavior and Spread of AIDS in Mexico », dans Ralph Bolton, The AIDS Pandemic, A Global Emergency, New York, Gordon and Breach, p. 37-50.

Cash, R.A. (1994), « Heterosexual Behavior Related to the Risk of HIV Infection among Japanese Men in Bangkok, Thailand ", International Conference on AIDS, 7-12 août, 10, 440, résumé $\mathrm{n}^{\circ} \mathrm{PD} 0367$.

Choi, Kyung Hee, Joe Catania, Thomas J. Coates, et Norman Hearst (1992), «International Travel and AIDS Risk in South Korea », International Conference on AIDS, 8 (2), résumé $n^{0}$ PoC 4624.

Clift, Stephen, et Simon Forrest (1999), « Gay Men and Tourism: Destinations and Holiday Motivations », Tourism Management, vol. 20, p. 615-625.

Cohen, Eric (1982), « Thai Girls and Farang Men: The Edge of Ambiguity », Annals of Tourism Research, vol. 9, p. 403-428.

Cohen, Eric (1971), « Arab Boys and Tourist Girls in a Mixed Jewish-Arab Community », International Journal of Comparative Sociology, vol. 12, p. 217-233.

Cohen, E., et E. Taylor (1976), « Environmental Orientations: A Multi-Dimensional Approach to Social Ecology », Current Anthropology, vol. 17, p. 49-70.

Dahles, Heidi, et Karin Bras (1999), " Entrepreneurs in Romance: Tourism in Indonesia », Annals of Tourism Research, vol. 26, p. 267-293.
Daniels, D., P. Kell, M. Nelson, et S. Barton (1992), « Sexual Behavior among Travellers: A Study of Genitourinary Medicine Clinic Attenders », International Journal of STD \& AIDS, vol. 3 .

Farley, Melissa, Isin Baral, Merab Kiremire, et Ufuk Sezin (1998), " Prostitution in Five Countries: Violence and Post-Traumatic Stress Disorder », Feminism and Psychology, vol. 8, p. 405-426.

Ford, N. (1991), Sex on Holiday: The HIVRelated Sexual Interaction of Young Tourists Visiting Torbay, University of Exeter, Exeter.

Ford, N. (1990), A Comparative of the SocialSexual Lifestyles amongst Travellers: A Study of Genitourinary Medicine Clinic with Other Young Residents in Devon and Cornwall, University of Exeter, Exeter.

Ford, K., D.N. Wirawan, P. Fajans, et L. Thorpe (1995), « AIDS Knowledge, Risk Behaviors, and Factors Related to Condom Use among Male Commercial Sex Workers and Male Tourist Clients in Bali, Indonesia », AIDS, vol. 9, p. 751-759.

Ford, N., et M. Inman (1992), « Safer Sex in Tourist Resorts », World Health Forum, vol. 13, p. 77-80.

Forsythe, Steven, Julia Hasbùn, et Martha Butler De Lister (1998), « Protecting Paradise: Tourism and AIDS in the Dominican Republic », Health Policy and Planning, vol. 13, p. 277-286.

Graburn, N. H (1983), « Tourism and Prostitution ", Annals of Tourism Research, vol. 10, p. 110-116.

Hall, C.M. (1992), « Sex Tourism in South-East Asia », dans D. Harrison (dir.), Tourism and the Less Developed Countries, Londres, Belhaven Press, p. 64-74.

Herold, Edward, Rafael Garcia, et Tony DeMoya (2001), «Female Tourists and Beach Boys: Romance or Sex Tourism? », Annals of Tourism Research, vol. 28, p. 978-997.

Kleiber, D., et M. Wilke (1995), « Sexual Behaviour of German (Sex)Tourists, International Conference on AIDS, 6-11 juin, 10(1), résumé n ${ }^{\circ}$ WS-D-10-2.

Krippendorf, Jost (1987), Les vacances et après ? : Pour une nouvelle compréhension des loisirs et des voyages, Paris, L'Harmattan.

Lett, J.W. (1983), « Ludic and Liminoid Aspects of Charter Yacht Tourism in the Caribbean ", Annals of Tourism Research, vol. 10, p. 35-56.

Lévy, Joseph J., Stéphanie Laporte, et Mansour El Feki (2001), « Tourisme et sexualité en Tunisie », Anthropologie et Sociétés, vol. 25, p. 61-68.
Manderson, Lenore (1992), « Public Sex Performances in Patpong and Explorations of the Edges of Imagination ", Journal of Sex Research, vol. 29, p. 451-475.

Maurer, Mechtild (1992), Tourisme, prostitution et sida, Paris, L'Harmattan.

Mayo, E.J., et L.P. Jarvis (1981), The Psychology of Leisure Travel, Massachusetts, CBI Publishing Company.

Nossa, P., P. Malta, M. Cruz, et J. Correia (1994), «AIDS and Tourism in a Sun, Sand and Sea Tourist Destination: The Portuguese Case », International Conference on AIDS, 712 août, 10(1), 443, résumé $n^{\mathrm{O}}$ PD0381.

O'Connell Davidson, Julia (2001), «The Sex Tourist, the Expatriate, His Ex-Wife and Her 'Other': The Politics of Loss, Difference and Desire », Sexualities, vol. 4, p. 5-24.

O'Malley, J. (1988) « Sex Tourism and Women's Status in Thailand », Loisir et Société, vol. 11, p. 99-114.

Oppermann, Martin (1999), "Sex Tourism », Annals of Tourism Research, vol. 26, p. 251-266.

Pritchard, Annette, et Nigel Morgan (2000), «Privileging the Male Gaze Gendered Tourism Landscapes? », Annals of Tourism Research, vol. 27, p. 884-905.

Pruitt, D., et S. Lafont, (1995), « For Love and Money: Romance Tourism in Jamaïca ». Annals of Tourism Research, vol. 22, $\mathrm{n}^{\circ}$ 2, p. $422-440$.

Rodriguez-Garcia, Rosalia (2001), « The Health-Development Link: Travel as a Public Health Issue », Journal of Community Health, vol. 26, p. 93-112.

Ryan, Chris (1991), Recreational Tourism: a Social Science Perspective, London, Routledge.

Ryan, Chris, et Amber Martin (2001), « Tourist and Strippers: Liminal Theater », Annals of Tourism Research, vol. 28, p. 140-163.

Vorakitphokatorn, Sarudee, et R. Cash (1992), « Factors that Determine Condom Use among Traditionally High Users: Japanese Men and Commercial Sex Workers in Bangkok, Thailand », International Conference on AIDS, 8(2), résumé $\mathrm{n}^{\circ}$ PoD 5237.

Vorakitphokatorn, Sairudee (1994), « Sexual Behavior of Young Japanese Women Tourists in Southern Thailand and Risk for HIV Infection », International Conference on AIDS, 7-12 août, 10(1), 23, résumé n 066D. 